\title{
Simulation of defects in bubble clusters
}

\author{
S.J. Cox ${ }^{1}$ P.I.C. Teixeira ${ }^{2,3}$ and M. Fátima Vaz ${ }^{4}$ \\ ${ }^{1}$ Institute of Mathematics and Physics, Aberystwyth University, Aberystwyth SY23 \\ 3BZ, United Kingdom \\ ${ }^{2}$ Instituto Superior de Engenharia de Lisboa, Rua Conselheiro Emídio Navarro 1, \\ P-1950-062 Lisboa, Portugal \\ ${ }^{3}$ Centro de Fisica Teórica e Computacional da Universidade de Lisboa, Avenida \\ Professor Gama Pinto 2, P-1649-003 Lisboa, Portugal \\ ${ }^{4}$ Instituto de Ciência e Engenharia de Materiais e Superfícies and Departamento de \\ Engenharia de Materiais, IST, UTL, Avenida Rovisco Pais, P-1049-001 Lisboa, \\ Portugal
}

\begin{abstract}
Topological defects in a foam, either isolated (disclinations and dislocations) or in pairs, affect the energy and stress, and play an important role in foam deformation. Surface Evolver simulations were performed on large finite clusters of bubbles. These allow us to evaluate the effect of the topology of the defects, and the distance between defects, on the energy and pressure of foam clusters of different sizes. The energy of such defects follows trends similar to known analytical results for a continuous medium.
\end{abstract}

PACS numbers: 47.57.Bc, 61.72.J-

Submitted to: J. Phys.: Condens. Matter

\section{Introduction}

Soft materials, such as aqueous foams, block copolymers and colloidal emulsions exhibit ordered structures which present a wide variety of complex geometries and topologies. These ordered structures are not perfect and may contain several types of defects.

Defects can be classified into two types: topological defects, which engender no change in area, and geometrical defects, which do. Dislocations and disclinations are included in first class, and it is these that we consider here. The study of defects is important because defects in the crystal structure are responsible for many of the physical, chemical and mechanical properties of a material. For example, the plastic deformation of metals occurs due to the motion of dislocations [1]. For elastoplastic materials, an extensive analysis has been made to find exact analytical solutions for all characteristic fields of screw and edge dislocations [2], wedge [3] and twist [4] disclinations. 
In two dimensions (2D), crystal structures are based upon the hexagonal lattice (or its dual, the triangular lattice). This perfectly ordered structure has been studied extensively, particularly in the context of 2D foams (i.e. monolayers of bubbles), but the situation in which the lattice contains a small number of topological defects has been rather neglected. Exceptions include experiments [5-7] and simulations [8], with an emphasis on determining the coarsening behaviour of the foam over time. In contrast, rather more authors consider single geometrical defects [9-12].

A 2D foam can be viewed as a cellular pattern for which the surface energy is the total perimeter multiplied by the value of surface tension (assumed here to be constant). The ordered state is hexagonal [13] and a disclination is thus a non-hexagonal bubble, while the elementary dislocation (i.e. that with the smallest Burgers vector) is a pair of 5- and 7-sided bubbles $[14,15]$. We next survey results for the strain energy of disclinations and dislocations before describing simulations of 2D foams containing them and comparing the two.

\section{Strain energy of disclinations and dislocations}

\subsection{Disclinations}

A useful way to think about disclinations in a solid is provided by the Volterra construction. Consider a torus of material lying in the $x y$-plane: disclinations are produced by inserting $(P>0)$ or removing $(P<0)$ a wedge of angle $P \pi / 3$ in the torus $[16,17]$, where we denote by $P$ the strength of the disclination. To accommodate the wedge, the torus has to be cut from the outer edge to the hollow core. Disclinations are classified according to the relative motion of the two cut surfaces: rotation around the axis of the torus will produce a wedge disclination [18], whereas rotations around axes perpendicular to the axis of the torus will produce twist disclinations. In what follows only 2D wedge disclinations are considered.

Disclinations are rarely observed in 3D structures such as metals because they are energetically very costly [18], but are seen occasionally in foams [6,7] and they often appear in block copolymers [19] and liquid crystals [16, 18, 20-22], particularly in nematic liquid crystals. The latter typically consist of elongated molecules that tend to be oriented in the same direction, but are positionally disordered. In a nematic liquid, the energy of a wedge disclination is

$$
w=\pi K P^{2} \ln \left(\frac{\rho}{a}\right),
$$

where $\rho$ is the distance between the dislocation line and the container, $a$ is the molecular dimension and $K$ is the average elastic constant [16].

In addition, disclinations may occur in pairs with opposite signs. They tend to be coupled at short distances since this reduces long-range distortion [20]. In a nematic liquid the energy per unit length of line, $w$, for two wedge disclinations of opposite strengths $+P$ and $-P$ a distance $d$ apart is

$$
w=2 \pi K P^{2} \ln \left(\frac{d}{a}\right) .
$$


Compared to equation (1) a factor of two is introduced and $\rho$ is replaced by the distance $d$ between the two defects $[16,20]$.

The strain field due to a disclination can be evaluated within an elastic continuum model that assumes a strain field with radial symmetry [23]. When applied to a hexagonal $2 \mathrm{D}$ foam, the strain energy density $w$, i.e. the energy per unit area per unit length, of a disclination cluster of strength $P$ was found to be

$$
w=\frac{G}{36} P^{2},
$$

where $G$ is the shear modulus [23]. For a given distance $r$ from the core to the external part of the defect, the strain energy is thus proportional to $P^{2}$, and independent of $r$. In a $2 \mathrm{D}$ incompressible medium, the elastic modulus $E$ satisfies $E=4 G$, and for a $2 \mathrm{D}$ hexagonal lattice

$$
G=\frac{1}{2 \sqrt{3}} \frac{\gamma}{a_{0}}
$$

where $a_{0}$ is the edge length of a hexagonal bubble with area $A$ (i.e. $A=\frac{1}{2} 3 \sqrt{3} a_{0}^{2}$ ) and $\gamma$ is the film tension [23].

\subsection{Dislocations}

Dislocations in a continuous medium can likewise be understood in terms of the Volterrra construction. The torus is cut again from the outer edge to the hollow core; any motion of the two cut surfaces that has no rotational component yields a dislocation. The motion can be in one of three directions: if it occurs along one of the two axes that are perpendicular to the axis of the torus, edge dislocations are obtained. If the motion is parallel to the axis of the torus, it defines a screw dislocation [18]. Only edge dislocations will be considered in this work.

Morral and Ashby [24] analysed dislocations in detail, including the pair of 5and 7-sided cells in a cellular structure. Dislocations in foams have been observed since the pioneering work of Bragg and Nye [25], who noted them in bubble rafts. 5/7 dislocations were also observed in other types of liquid foams [26] and are responsible for the propagation of plastic deformation in foams [26, 27].

The energy of a dislocation can be described by the well-known equations of the elastic strain field of an edge dislocation [23, 28-30]. The strain energy density $w$ of a dislocation with Burgers vector $B$ in an incompressible foam cluster is

$$
w=\frac{G}{4 \pi} B^{2} \frac{1}{r^{2}} \text {. }
$$

Note that $w$ decreases with the distance $r$ from the core as $r^{-2}[23] \ddagger$.

The interaction energy of two edge dislocations with opposite signs a distance $d$ apart in the same glide plane can be adapted from [28, 29]:

$$
w=\frac{G}{\pi}\left(\frac{B}{\sqrt{A}}\right)^{2} \ln \left(\frac{d}{\sqrt{A}}\right) .
$$

$\ddagger$ We believe the factor of $\pi^{2}$ in the denominator of equation (21) in [23] to be in error. 


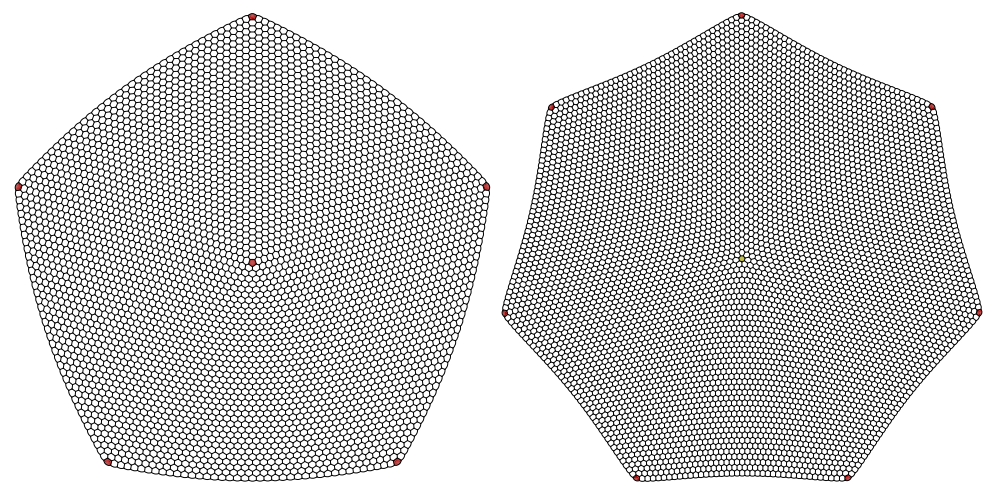

Figure 1. Examples of clusters with isolated disclinations for $n=5(N=4101, s=40$ shells) and $n=7(N=5741, s=40$ shells $)$.

When two dislocations of opposite signs are in the same glide plane, they attract each other to reduce their total elastic energy. That is, the dislocations act as dipoles and the strain energy changes with $\ln (d)$ and tends to zero as $d$ approaches the bubble size from above. Any closer, and they will combine and annihilate each other.

In the present work we study the effect of the presence of isolated defects, both dislocations and disclinations, on the energy of a 2D cluster, and the interaction of pairs of defects.

\section{Simulation method}

We simulate large polygonal foam clusters [31], consisting of $N$ bubbles of unit area, almost all of which are hexagonal. The clusters consist of a central bubble with $n$ sides surrounded by $s$ shells of hexagonal bubbles, and therefore have $n$-fold symmetry. In addition to the central defect, there are $n$ four-sided bubbles at the outer "corners" of the cluster. All internal bubbles have six neighbours, but due to the strain in the foam induced by the defects the bubbles neighbouring the defects are no longer regular hexagons.

The clusters are constructed from a Voronoi partition of an array of points. Defects are introduced by removing points from an ordered array. The resulting partition is then imported into the Surface Evolver [32] and the surface energy $E$ (equivalent to total perimeter) is minimized.

An isolated disclination in a cluster was formed by creating a central bubble with $n$ sides $(n \neq 6)$, surrounded by $s$ shells of hexagonal bubbles. We define the strength of the disclination to be $P=n-6$. Examples are shown for $n=5$ and $n=7$ disclinations in figure 1. Disclinations with $n=8$ and 9 were also considered.

Clusters containing pairs of disclinations were formed by joining two of these clusters, containing central bubbles with $n_{1}$ and $n_{2}$ sides, and eliminating a certain number of bubbles between the two disclinations to vary the separation $d$ between their centres. This introduces two seven-sided bubbles at the periphery of a cluster, one at each end of the join, which we presume does not change the interaction between 


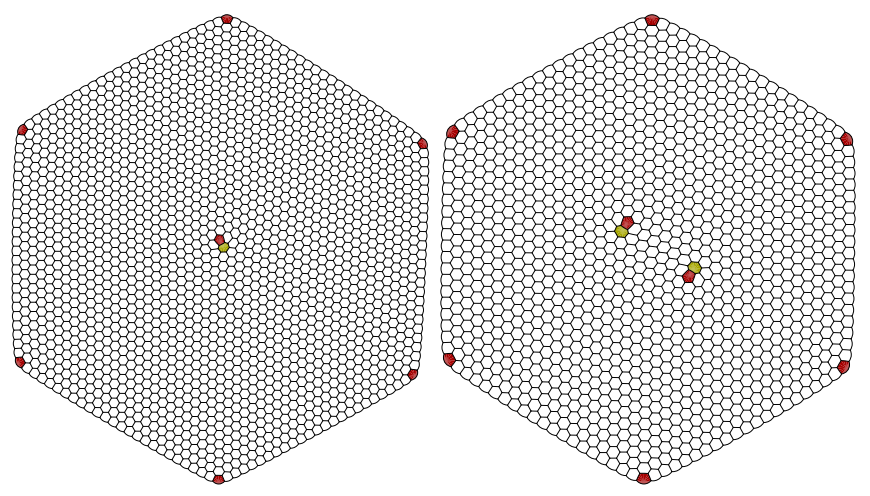

Figure 2. Examples of clusters with a single $5 / 7$ dislocation $(N=1925, s=25$ shells $)$ and a pair of $5 / 7$ dislocations ( $N=1261, s=20$ shells) in a six-fold symmetric cluster.

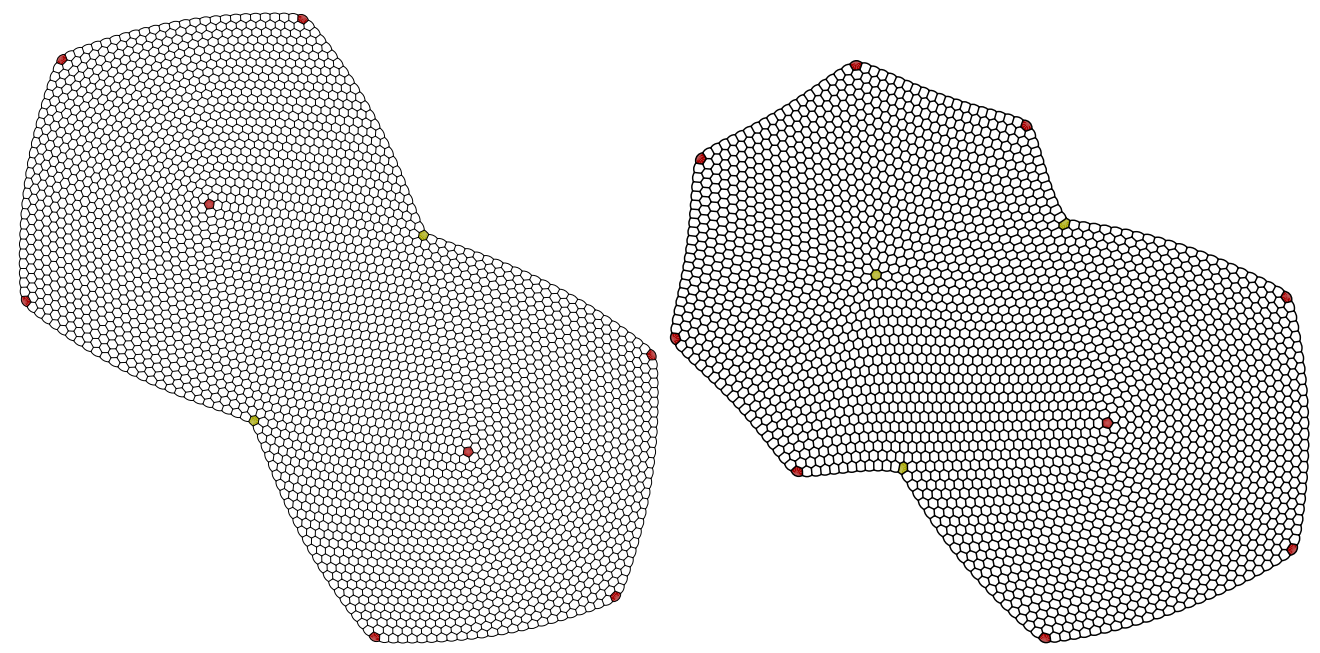

Figure 3. Examples of clusters with pairs of separated disclinations for $n_{1}=n_{2}=$ $5(N=3252, s=25$ shells, $d \approx 42)$ and $n_{1}=5, n_{2}=7(N=2740, s \approx 25$ shells, $d \approx 30)$.

the two centralized defects. We considered pairs of disclinations of the same strength ( $n_{1}=n_{2}=5$ or 7 ) or opposite $\left(n_{1}=5\right.$ and $n_{2}=7$ ). Examples are shown in figure 3 .

A $5 / 7$ dislocation is constructed from a hexagonal cluster $(n=6)$ by removing a wedge of bubbles originating at the centre (figure 2). To simulate pairs of dislocations we again start from a hexagonal cluster and perform a single topological change on an edge near the centre [15]. This allows us to study the special case in which the two dislocations have opposite sign and are in the same glide plane (figure 2). To change the distance between the defects, we perform further topological changes. The Burgers vector of a dislocation is the vector between adjacent hexagons, parallel to the $5 / 7$ edge, which defines the direction of gliding. The two dislocations of figure 2 have opposite Burgers vectors, i.e. have opposite signs, but share the same glide plane. 


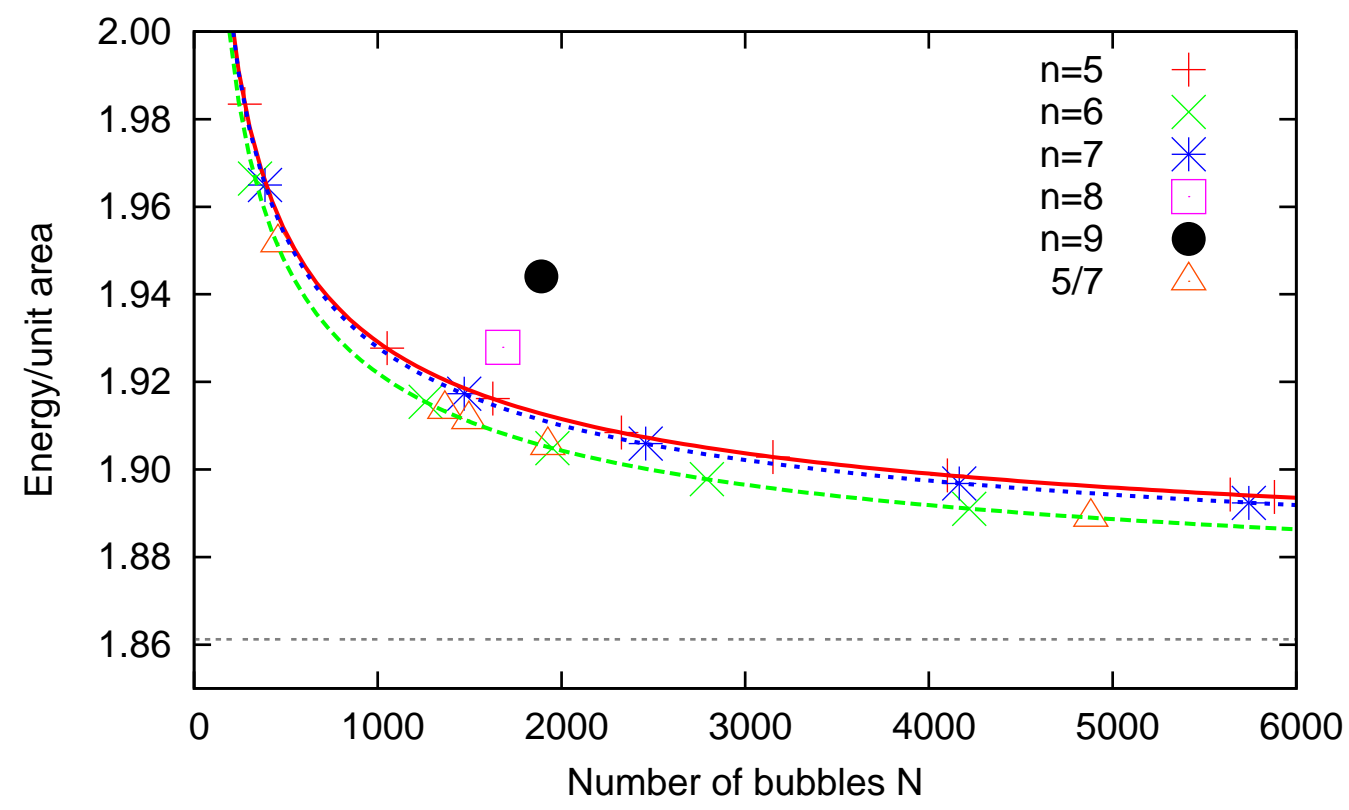

(a)

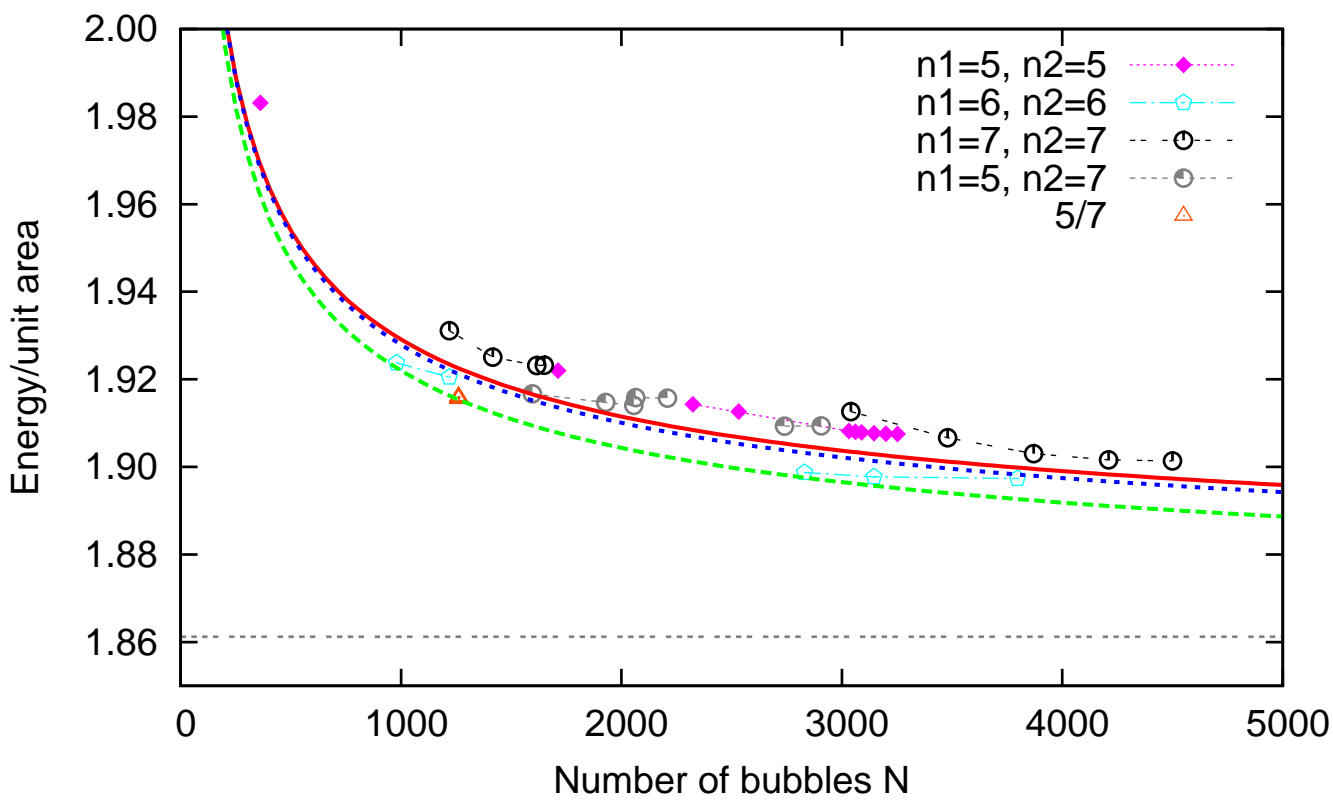

Figure 4. Energy per unit area, $\hat{E}$, for all clusters. (a) Clusters with isolated disclinations for $n=5$ to 9 ; the lines are fits to the form $N^{-1 / 2}$ for $n=5,6,7$, and the horizontal line is half the perimeter of a regular hexagon of unit area. Also shown are clusters with 5/7 dislocations. (b) Clusters with pairs of defects separated by different distances $d$. The continuous lines are the fits shown in (a). Points joined by lines are for the same number of shells $s$. The energy of a pair of dislocations varies relatively little with $d$ and is shown as a single point.

\section{Results}

\subsection{Disclinations}

The energy (or perimeter) per unit area (i.e. per bubble), $\hat{E}=E / N$, decreases as the number of shells (bubbles) increases for given $n$, as shown in figure 4(a). For given 
$s, \hat{E}$ is least for $n=6$. In fact, the data is described very well by the following two-parameter fits:

$$
\begin{aligned}
& \hat{E}_{5}(N)=1.86898+1.90172 N^{-1 / 2}, \\
& \hat{E}_{6}(N)=1.86171+1.90625 N^{-1 / 2}, \\
& \hat{E}_{7}(N)=1.86701+1.92538 N^{-1 / 2},
\end{aligned}
$$

which are also shown in figure 4(a). Note the value 1.86 that recurs - it is close to half the perimeter of a regular hexagon of unit area in an infinite honeycomb, since each side is shared between two bubbles. This means that the second term of each expression for the energy can be regarded as an excess energy that reflects the effect of the topology of the defect on the external boundary of the cluster, as will be discussed.

The energy of a bubble cluster can be expressed as a sum of the bubble areas $A_{i}$ and pressures $p_{i}$ [33]: $E=2 \sum A_{i} p_{i}$, relative to an external pressure taken as zero. In the monodisperse case considered here, we therefore find that the average pressure is $\langle p\rangle=\frac{1}{2} \hat{E}$, so that the average pressure in a cluster containing a single disclination decreases in the manner shown in figure 4(a).

The individual bubble pressures vary significantly about the average, however. The pressure of the central bubble is strongly correlated with $n$, and varies only weakly with $N$ (figure 5(a)). For $n \leq 5, P \leq-1$ the bubble pressure is highest at the centre of the cluster, while for $n \geq 7, P \geq+1$ it is higher at the periphery, as can be seen in figure $5(b-c)$.

The energy per unit area in a cluster containing two disclinations is shown in figure 4(b). It decreases in much the same way as for clusters containing a single disclination, showing a similar dependence on the cluster size, $N$.

\subsection{Dislocations}

The energy per unit area in an approximately six-fold cluster with a 5/7 defect at the centre is shown in figure 4(a). The energies of this type of defect are close to the value for a defect-free (hexagonal) cluster, suggesting that the 5/7 dislocation has a lesser cost than a disclination as would be the case in a solid crystal.

This is confirmed by the energy of a pair of dislocations a distance $d$ apart (figure 4(b)): the dependence on $d$ is not significant compared to the dependence on $N$, and the energy/unit area is close to the value for a defect-free cluster.

The pressure distribution in a dislocation cluster is shown in figure 5(d): the average pressure in each shell is much the same as for a defect-free cluster $(n=6)$, but the scatter in pressures increases to a maximum close to the centre of the cluster. The two become coincident about 15 shells from the centre of the cluster, giving a measure of the "screening length" [34]. 
(a)

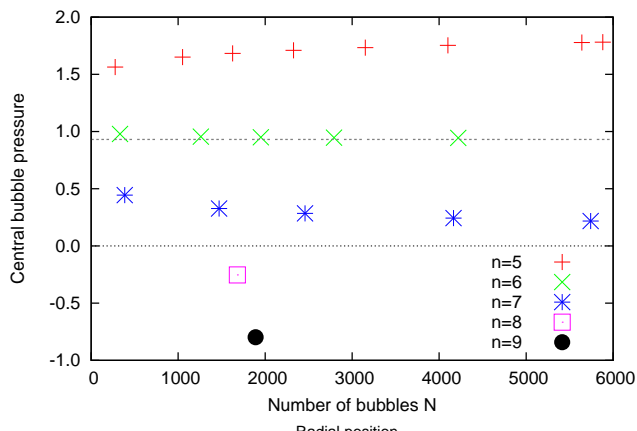

(c)

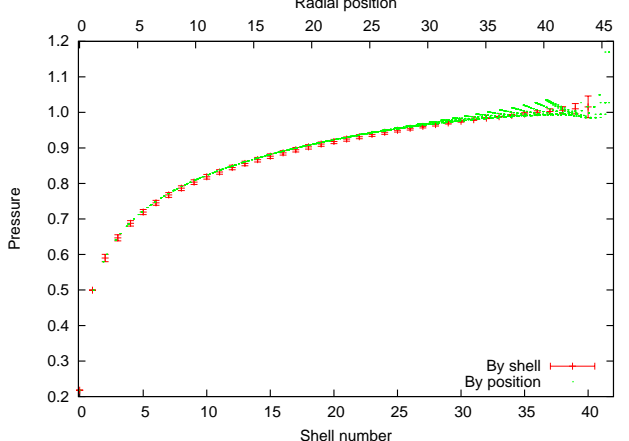

(b)
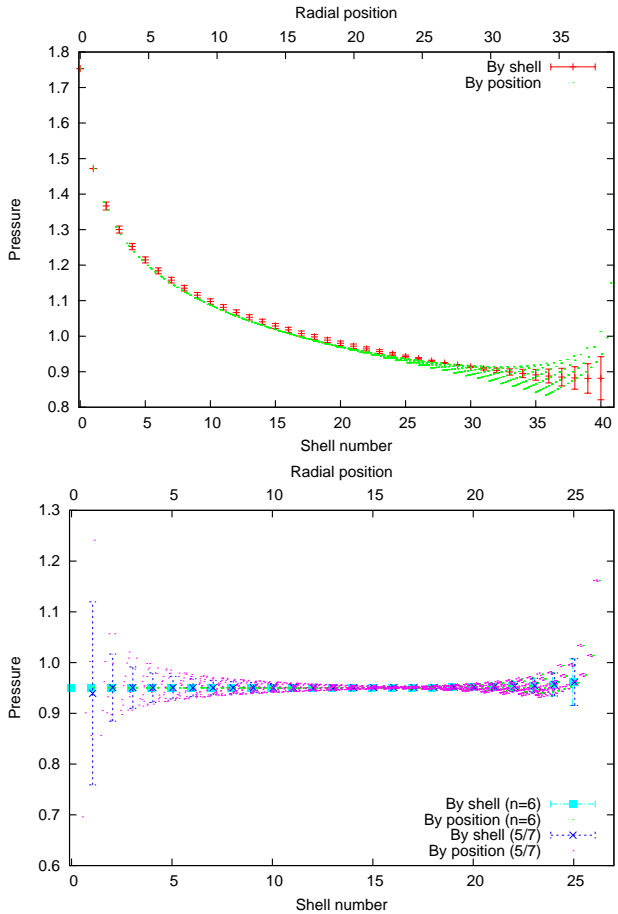

Figure 5. (a) Pressure in the central bubble of a cluster containing a single disclination. The pressure depends strongly on the value of $n$, but weakly on the size of the cluster. (b-d) Bubble pressure as a function of radial distance from the centre of the cluster, and the average pressure in each shell (error bars show standard deviation) for clusters with (b) $n=5$ disclination, $s=40, N=4101$, (c) $n=7$ disclination, $s=40, N=5741$, (d) $n=6$ disclination, $s=25, N=1951$ and 5/7 dislocation, $s=25, N=1926$. Notice the change of slope for $n=5$ versus $n=7$ and that the dislocation introduces only a local perturbation of the pressures compared to the hexagonal case.

\section{Discussion}

\subsection{Disclinations}

The variation of $\hat{E}$ with $N$ may be interpreted as follows. Exact calculations for clusters with disclinations and different areas predict that the energy of a cluster is [35]

$$
E=\frac{3.72}{2} \sum_{i} A_{i}^{1 / 2}+2.04\left(\sum_{i} A_{i}\right)^{1 / 2},
$$

in terms of the bubble areas $A_{i}$. The second term of the equation is related to the shape of the cluster boundary, and 2.04 corresponds to a rounded cluster. For clusters with regular hexagonal boundary the factor 2.04 decreases to 1.94 [35]. For clusters with unit area this leads to

$$
\hat{E}=\frac{E}{N}=1.86+1.94 N^{-1 / 2}
$$

This is in accordance with our findings ( $\$ 4.1$, equation (8) and figure 4), although it does not distinguish between clusters with different $n$, i.e. it does not take topology 


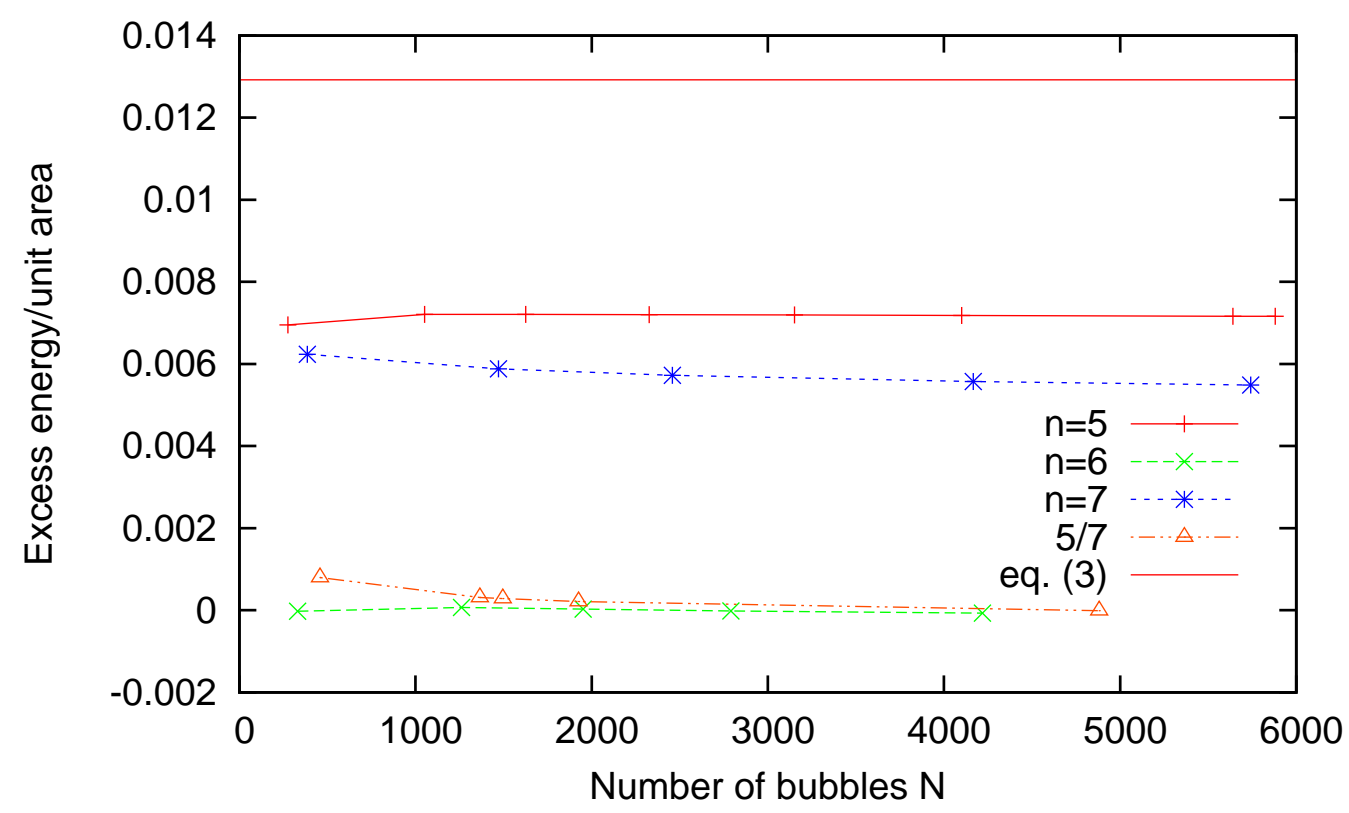

(a)

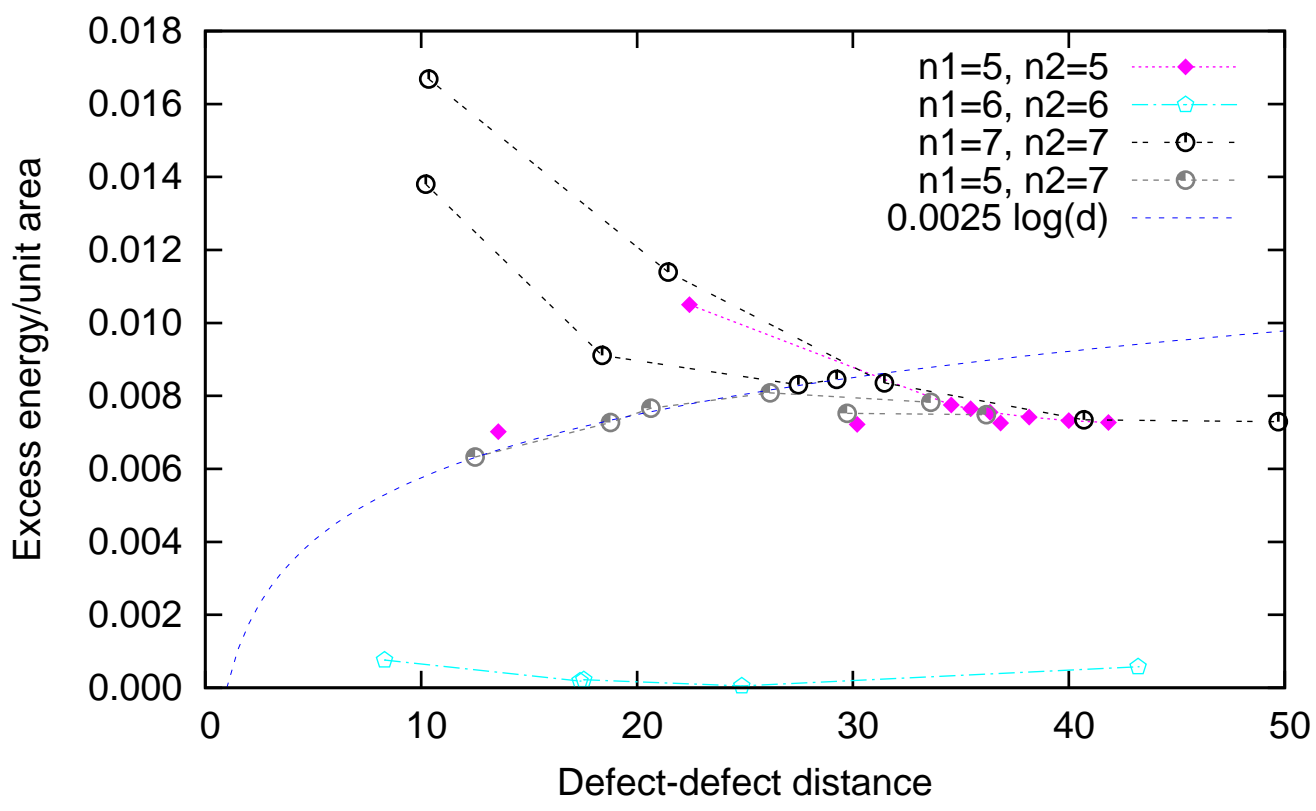

Figure 6. (a) Excess (strain) energy $w=\hat{E}-\hat{E}_{6}(N)$, for all clusters with isolated disclinations for $n=5$ to 7 and a $5 / 7$ dislocation. The horizontal line is the value given by equation (3) for bubbles of unit area. (b) Excess (strain) energy $w=\hat{E}-\hat{E}_{6}\left(N^{\prime}\right)$ for clusters with pairs of disclinations. Points joined by lines are for the same number of shells $s$. Also shown is a logarithmic fit to the data for $n_{1}=5, n_{2}=7, s=19$. All data converge to the values for a single disclination, shown in (a).

into account.

For isolated disclinations of strength $P$ in a 2D foam the energy density should be determined by equation (3). This is the excess energy density relative to the energy of a perfect hexagonal $2 \mathrm{D}$ foam $(w=0$ if $P=0)$. In order to compare the energy of 
isolated defects in foams, we propose to calculate $w$ as

$$
w=\hat{E}-\hat{E}_{6}(N),
$$

where $\hat{E}$ is the total perimeter per unit area obtained in the simulations, see figure $6($ a). Equation (3) implies that the energy density for an $n=5$ disclination $(P=1)$ is the same as for an $n=7$ disclination $(P=+1)$, for the same radius, which is indeed seen to be approximately the case, but the magnitude of $w$ is only about half of that predicted.

For paired disclinations a distance $d$ apart, we define the strain (excess) energy in a similar way by subtracting the energy of a joined cluster without defects. The number of rows of bubbles that are removed when the clusters are joined varies as $s-d$, where $s$ is the number of shells. We therefore propose the following expression:

$$
w=\hat{E}-\hat{E}_{6}\left(N^{\prime}\right) \text { with } N^{\prime}=N+k_{1} s\left(k_{2} s-d\right)
$$

where $k_{1}$ and $k_{2}$ are two fitting parameters extracted from the case $n_{1}=n_{2}=6$; we find $k_{1}=1.1$ and $k_{2}=0.5$. This should take into account the shape of two joined clusters and the presence of two extra defects at the end of the line where they join.

Figure 6(b) shows that $n_{1}=n_{2}=5$ and $n_{1}=n_{2}=7$ have similar strain energies, which decrease, as expected, as the separation of the like defects becomes greater.

The energy density of two disclinations of opposite strengths in a nematic liquid crystal is predicted to depend on their separation $d$ through equation (2). We hypothesize that the energy density of two disclinations in a 2D foam has a similar functional form: assuming that the defect core is the size of the central bubble $A$, we take $a=\sqrt{A}=1$ and write

$$
w=M P^{2} \ln \left(\frac{d}{\sqrt{A}}\right),
$$

where $M$ is an elastic constant. The case $n_{1}=5, n_{2}=7$, shown in figure 6(b), certainly increases with $d$, and a logarithmic fit with $M=2.5 \times 10^{-3}$ seems reasonable.

\subsection{Dislocations}

The Burgers vector of the dislocation of figure 2 can be related to $a_{0}$, the edge length of a bubble with area $A$, to give $B=\sqrt{3} a_{0}$, which is the smallest possible component of the vector in a hexagonal cell, equivalent to the centre-to-centre distance between bubbles. Thus for a hexagonal foam containing bubbles of unit area we have $B=1.074$. Equation (5) gives the energy density of a dislocation cluster of size $r$ in an infinite honeycomb. Although it was derived for a cylindrical body, we take $r$ as the radius of a circle with perimeter equal to the perimeter $L$ of the periphery of the cluster, so that $r \sim \sqrt{N}$.

Figure 7 shows that the energy density decreases with the radial size of the cluster as $1 / r^{\alpha}$ with $\alpha=1.88 \pm 0.24$; this is not far from $\alpha=2$ as predicted by equation (5), although the prefactor appears to be out by a factor of 4 .

For paired dislocations a distance $d$ apart (figure 2, right), we calculate the strain energy by subtracting the energy of the same (hexagonal) cluster without defects, 


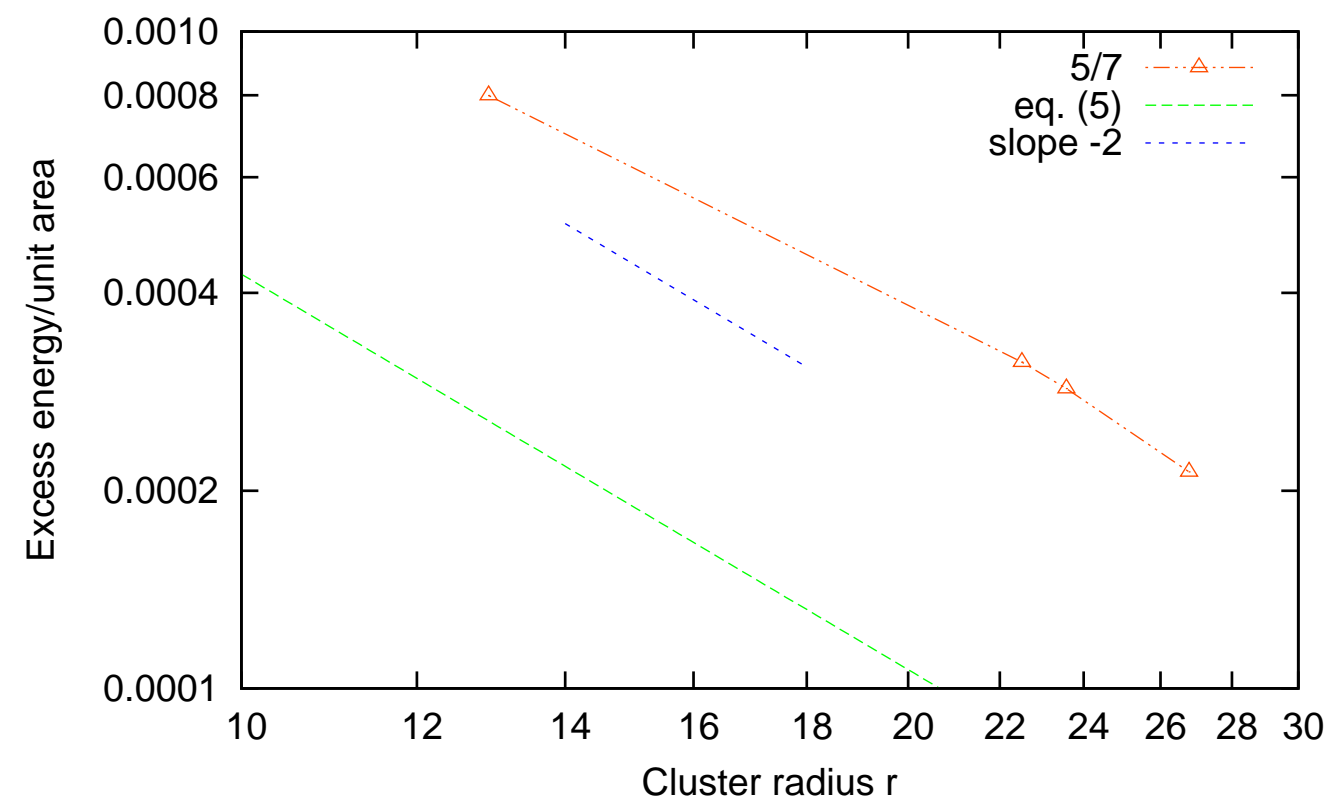

(a)

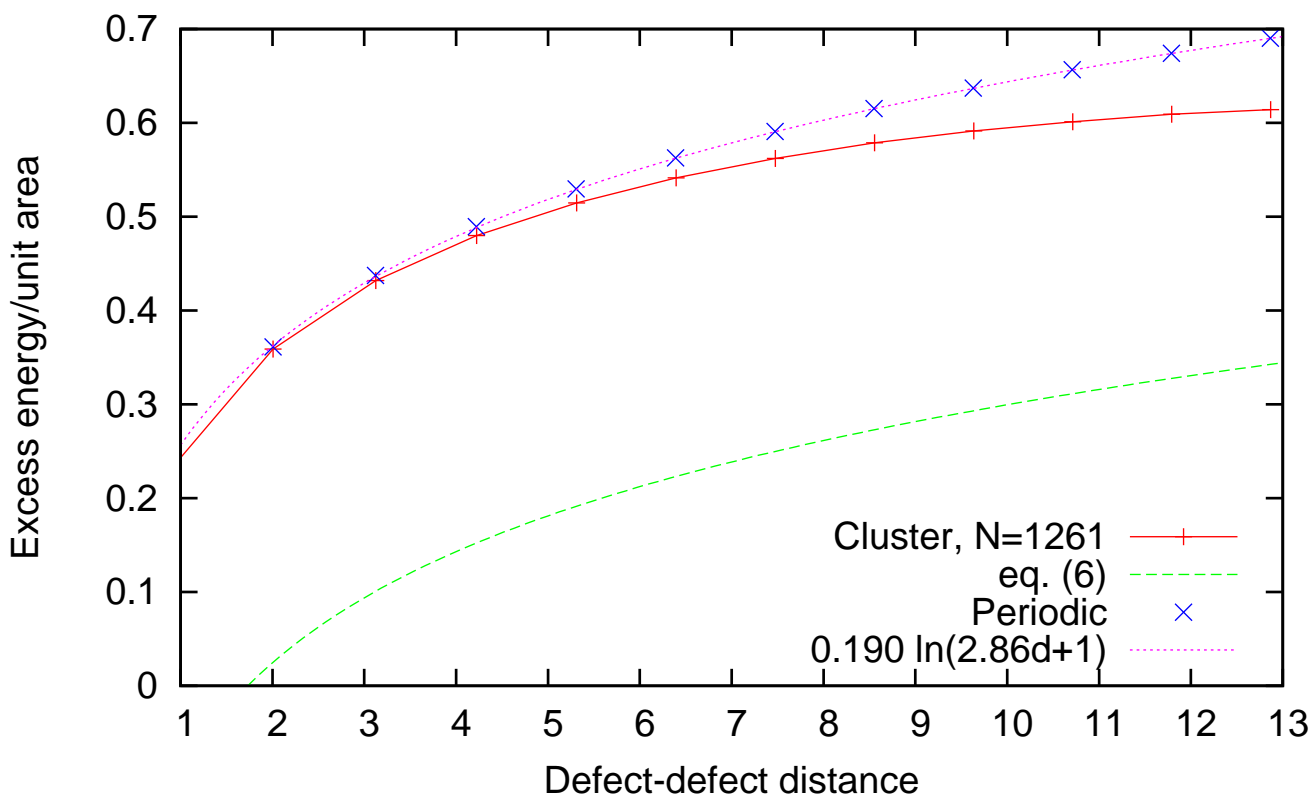

Figure 7. (a) Excess (strain) energy per unit area, $\hat{E}-\hat{E}_{6}(N)$, for a $5 / 7$ dislocation. The data fit to $r^{-\alpha}$ with $\alpha=1.88 \pm 0.24$, which is consistent with the functional dependence given by equation (5). (b) Excess energy per unit area for two 5/7 dislocations a distance $d$ apart. The result for a finite 6-fold cluster of $N=1261$ bubbles is compared with the prediction of equation (6) and the result for an infinite honeycomb and its logarithmic fit. 
shown in figure 7(b). The strain energy increases almost logarithmically with $d$, according to (6), shown in figure 7(b). In this case it is possible to compare the data with the same defects embedded in an infinite (periodic) hexagonal foam, eliminating the effect of the cluster boundaries. The a posteriori confirmation that the boundaries do not have a large effect on the interaction between defects is apparent from the proximity of the lines in figure 7(b). The strain energy in the hexagonal foam is fit better by a function of the form $\ln (k d+1)$

\section{Conclusion}

This work describes simulations of 2D finite aqueous foam clusters containing topological defects. The presence of one defect, in particular a disclination, clearly affects the energy and the pressure of the cluster. The energy of a disclination cluster deviates from the energy of a defect-free cluster if the number of sides of the central cell is different from six, and decreases as the number of shells increases. Both the energy and the pressure of a cluster with $n=6$ match very well with those found for a 5/7 dislocation cluster. In fact, the average pressure in each shell is the same in the two cases.

Many processes, such as plastic deformation, deal with the interactions between defects. Our simulations of pairs of defects reveal how the presence of one defect is "felt" by the other defect as a function of their separation. Analytic approaches have been developed, in the context of solids or of liquid crystals, for the same cases as studied here. For most of them defects in foams follow the predicted trends. For example, the energy of two disclinations with opposite strengths a distance $d$ apart appears to be proportional to $\ln d$.

Nonetheless, a perfect match between analytical results and simulations is not to be expected, as the assumptions under which the former were derived are not always satisfied in the systems considered here. Clusters with $n=5$ and $n=7$ are examples of this: because these disclinations have the same strength (in absolute value), one would expect that they would have the same energy, yet the two clusters have different boundaries, and it is clear that the energy of a cluster is strongly dependent on boundary shape. Further work still needs to be undertaken to fully separate the contributions of defect shape and cluster shape to the energy of a foam.

\section{Acknowledgments}

We acknowledge support from the British Council/FCT Transnational Cooperation Scheme. SJC thanks G. Mishuris, A. Mughal and A. Piccolroaz for useful discussions, and EPSRC for funding (EP/D071127/1, EP/D048397/1).

\section{References}

[1] A. Cottrell, Mechanical Properties of Matter (Wiley, New York, 1964). 
[2] M. Lazar, Comp. Mat. Sci. 28, 419 (2003).

[3] M. Lazar, Phys. Lett. A 311, 416 (2003).

[4] M. Lazar, J. Phys.: Condens. Matter 15, 6781 (2003).

[5] A. Abd-el-Kader and J.C. Earnshaw, Phys. Rev. E 58, 760 (1998).

[6] Y. Ishida and S. Iyama, Acta Metallurgica 24, 417 (1976).

[7] M. J. Bowick, L. Giomi, H. Shin and C.K. Thomas, Phys. Rev. E 77, 021602. (2008).

[8] H. J. Ruskin and Y. Feng, J. Phys.: Condens. Matter 7, L553 (1995).

[9] M. F. Vaz and M. A. Fortes, J. Phys.: Condens. Matter 9, 8921 (1997).

[10] W. Y. Tam, Phys. Rev. E 58, 8032 (1999).

[11] Y. Jiang, J. C. M. Mombach and J. A. Glazier, Phys. Rev. E 52 R3333 (1995).

[12] B. S. Gardiner, B. Z. Dlugogorski and G. J. Jameson J. Phys.: Condens. Matter 11, 5437 (1999).

[13] T. Hales, Discrete Comput. Geom. 25, 1 (2001).

[14] N. Rivier, M. F. Miri and C. Oguey, Coll. Surf. A: Phys. Eng. Aspects 263, 39 (2005).

[15] D. Weaire and N. Rivier, Contemp. Phys. 25, 59 (1984), reprinted in Contemp. Phys. 50, 199 (2009).

[16] P. G. de Gennes and J. Prost, The Physics of Liquid Crystals, 2nd Edition (Oxford University Press, Oxford, 1995).

[17] P. M. Chaikin and T. C. Lubensky, Principles of Condensed Matter Physics (Cambridge University Press, Cambridge, 1995).

[18] W. F. Harris, Scientific American 237, 130 (1977).

[19] R. A. Segalman, A. Hexemer, R. C. Hayward and E. J. Kramer, Macromol. 36, 3272 (2003).

[20] F. R. N. Nabarro (ed), "Other effects of Dislocations: Disclinations" in Dislocations in Solids, vol. 5, (North Holland, Amsterdam, 1980).

[21] M. Ravnik and S. Žumer, Soft Matter 5, 269 (2009).

[22] W. Song, H. Tu, G. Goldbeck-Wood and A. H. Windle, J. Phys. Chem. B, 109, 19234 (2005).

[23] M. A. Fortes and M. F. Vaz, J. Phys.: Condens. Matter 10, 7519 (1998).

[24] J. E. Morral and M. F. Ashby, Acta Metall. 22, 567 (1974).

[25] W. L. Bragg and J. F. Nye, Proc. Roy. Soc. Lond. A 190, 474 (1947).

[26] M. E. Rosa and M. A. Fortes, Phil. Mag. A 77, 1426 (1998).

[27] M. A. Fortes, M. E. Rosa and L. Afonso, Materials Science Forum 455-456, 644 (2004).

[28] F. R. N. Nabarro, Theory of Crystal Dislocations (Clarendon Press, Oxford, 1967). 
[29] D. Hull, Introduction to dislocations (Pergamon Press, Oxford, 1965).

[30] E. Clouet, Phil. Mag. 89, 1565 (2009).

[31] S. J. Cox and F. Graner, Phil. Mag. 83,2573 (2003).

[32] K. Brakke, Exp. Math. 1, $141 \quad$ (1992) (see also http://www.susqu.edu/brakke/evolver/).

[33] S. Ross. Ind. Engng. Chem. 61, 48 (1969).

[34] S. J. Cox, F. Graner and M. F. Vaz, Soft Matter 4, 1871 (2008).

[35] M. F. Vaz, M. A. Fortes and F. Graner, Phil. Mag. Lett. 82, 575 (2002). 